\section{Citric acid cycle intermediates as ligands for orphan G-protein-coupled receptors}

Weihai $\mathrm{He}^{1 \star}$, Frederick J.-P. Miao ${ }^{1}$, Daniel C.-H. Lin ${ }^{1}$, Ralf T. Schwandner ${ }^{1,2}$, Zhulun Wang ${ }^{1}$, Jinhai Gao ${ }^{1 \star}$, Jin-Long Chen ${ }^{1}$, Hui Tian ${ }^{1}$ \& Lei Ling ${ }^{1}$

${ }^{1}$ Tularik Inc., 1120 Veterans Boulevard, South San Francisco, California 94080, USA

${ }^{2}$ Tularik GmbH, Josef-Engert-Strasse 9, 93053 Regensburg, Germany

* Present addresses: Genospectra Inc., 6519 Dumbarton Circle, Fremont, California 94555, USA (W.H.); Novartis Institute of Biomedical Research Inc., 100 Technology Square, Cambridge, Massachusetts 02139, USA (J.G.)

The citric acid cycle is central to the regulation of energy homeostasis and cell metabolism ${ }^{1}$. Mutations in enzymes that catalyse steps in the citric acid cycle result in human diseases with various clinical presentations ${ }^{2}$. The intermediates of the citric acid cycle are present at micromolar concentration in blood and are regulated by respiration, metabolism and renal reabsorption/ extrusion. Here we show that GPR91 (ref. 3), a previously orphan G-protein-coupled receptor (GPCR), functions as a receptor for the citric acid cycle intermediate succinate. We also report that GPR99 (ref. 4), a close relative of GPR91, responds to $\alpha$-ketoglutarate, another intermediate in the citric acid cycle. Thus by acting as ligands for GPCRs, succinate and $\alpha$-ketoglutarate are found to have unexpected signalling functions beyond their traditional roles. Furthermore, we show that succinate increases blood pressure in animals. The succinate-induced hypertensive effect involves the renin-angiotensin system and is abolished in GPR91-deficient mice. Our results indicate a possible role for GPR91 in renovascular hypertension, a disease closely linked to atherosclerosis, diabetes and renal failure ${ }^{5,6}$.
In a search for natural ligands for orphan GPCRs, we tested extracts from various animal tissues for their ability to evoke an increase in intracellular $\mathrm{Ca}^{2+}$ concentration $\left(\left[\mathrm{Ca}^{2+}\right]_{\mathrm{i}}\right)$ using the aequorin assay ${ }^{7}$. We found that fractions from pig kidney extracts specifically activated cells expressing GPR91 (Fig. 1a). GPR91 is an orphan GPCR highly expressed in the kidney and shares 33\% amino acid identity with GPR99/GPR80 (refs 4, 8). On the basis of their homology with the purinergic receptor P2Y1, nucleotide ligands were predicted for GPR91 and GPR99 (ref. 4). However, the GPR91 ligand activity in pig kidney extracts was resistant to various stringent treatments including alkaline phosphatase, peptidase, and hydrolysis in $6 \mathrm{M} \mathrm{HCl}$ at $100^{\circ} \mathrm{C}$. Accordingly, the supposition that GPR91 might be activated by a nucleotide or peptide ligand was unlikely. We purified the natural ligand for GPR91 by ion-exchange, size-exclusion and reversed-phase fast performance liquid chromatography/high-performance liquid chromatography (Fig. 1a).

A major molecular ion $[\mathrm{M}+\mathrm{H}]^{+}$at $m / z 119.2$ was observed by mass spectrometry (Fig. 1b). ${ }^{1} \mathrm{H}$ NMR analysis revealed a single type of proton in the highly purified GPR91 ligand (Fig. 1c). ${ }^{13} \mathrm{C}$ NMR analysis further suggested the presence of $-\mathrm{CH}_{2}$ - (methylene) and $=\mathrm{C}=\mathrm{O}$ (carbonyl) groups (Fig. 1d). Combined with mass spectrometry results and the biochemical properties of the ligand, the purified GPR91 ligand was predicted and confirmed to be succinic acid (Fig. 1c, d).

Commercially obtained succinate (the physiological form of succinic acid) increased $\left[\mathrm{Ca}^{2+}\right]_{\mathrm{i}}$ dose-dependently in the aequorin assay (Fig. 2a). Succinate also activated mouse and rat orthologues of GPR91 (Fig. 2a). The succinate-induced increase in $\left[\mathrm{Ca}^{2+}\right]_{i}$ was further confirmed with a fluorimetric imaging plate reader (FLIPR) system in the 293-hGPR91 cell line (293 cells stably expressing human GPR91) (Fig. 2b). The concentration giving half-maximal response $\left(\mathrm{EC}_{50}\right)$ for succinate-induced activation of human GPR91 was $56 \pm 8 \mu \mathrm{M}$ in the aequorin assay and $28 \pm 5 \mu \mathrm{M}$ in the FLIPR assay. GPR91 was selectively activated by succinate but not by other citric acid cycle intermediates (Supplementary Information) or any of a collection of 800 pharmacologically active compounds and known GPCR ligands tested (data not shown). Furthermore, of 200

a

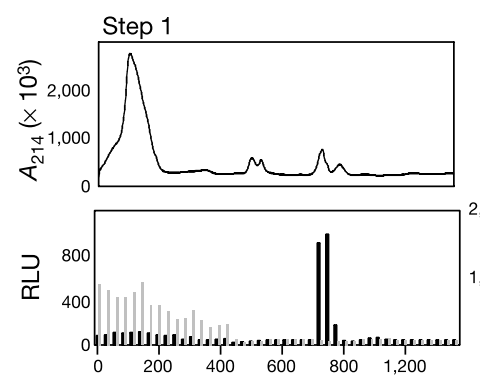

b

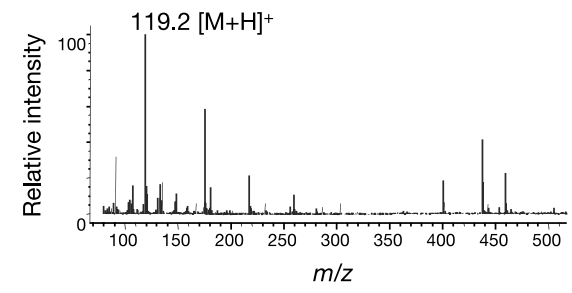

Figure 1 Purification and identification of GPR91 ligand from pig kidney extracts. a, Purification of GPR91 ligand through four steps of fast performance liquid chromatography/high-performance liquid chromatography (step 1, anion exchange; step 2 , size exclusion; step 3 , size exclusion; step 4 , reversed phase). The activity of each

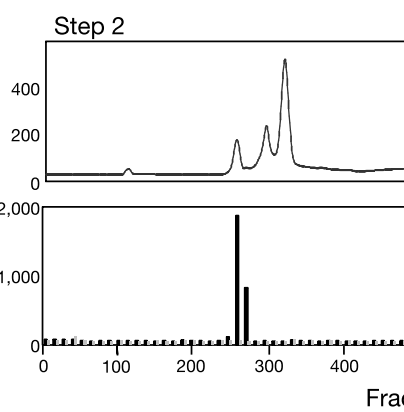

c

Purified GPR91 ligand

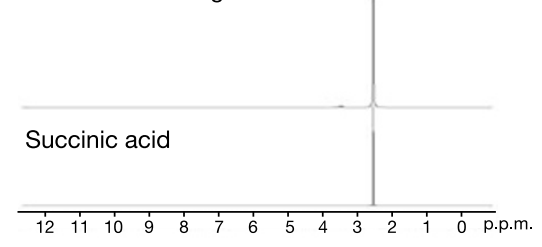

Step 3
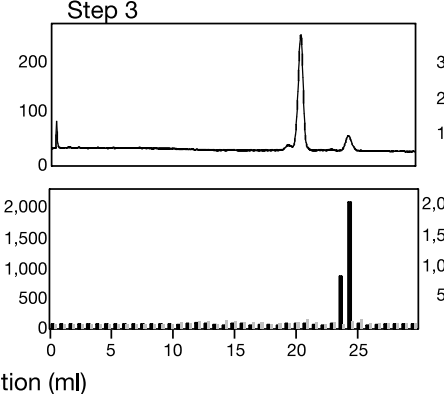
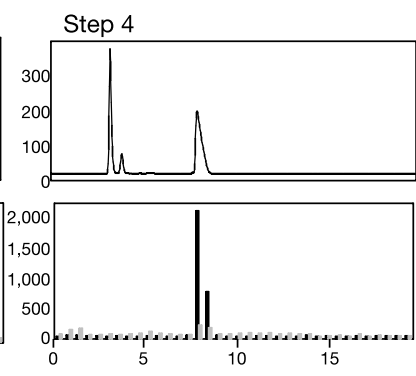

d

Purified GPR91 ligand 
carboxylic acids and structurally related analogues, succinate was found to be the most potent in increasing $\left[\mathrm{Ca}^{2+}\right]_{\mathrm{i}}$ (a representative subset is summarized in Supplementary Information).

Because GPR99 is the closest homologue of GPR91, we proposed that other citric acid cycle intermediates and carboxylic acids might activate GPR99. We identified $\alpha$-ketoglutarate as a ligand for human GPR99, with an $\mathrm{EC}_{50}$ of $69 \pm 11 \mu \mathrm{M}$ in the aequorin assay (Fig. 2c) and $32 \pm 4 \mu \mathrm{M}$ in the FLIPR assay (Fig. $2 \mathrm{~d}$ ). $\alpha$-Ketoglutarate also activated mouse GPR99 (Fig. 2c). Neither succinate nor $\alpha$-ketoglutarate activated calcium mobilization with about 30 GPCRs unrelated to GPR91 and GPR99 (data not shown).

To dissect the signalling pathways of GPR91 and GPR99, various biochemical assays were investigated. Succinate inhibited forskolinstimulated cAMP production in 293-hGPR91 cells, an effect abolished by preincubation with pertussis toxin (PTX) (Fig. 2e). In addition, succinate induced the accumulation of inositol phosphate in 293-hGPR91 cells (Fig. 2f). The succinate-induced accumulation of inositol phosphate and an increase in $\left[\mathrm{Ca}^{2+}\right]_{i}$ were each partly inhibited by pertussis toxin (Fig. $2 \mathrm{f}$ and Supplementary Information). Moreover, succinate activated extracellular signal-regulated kinase (Erk) in 293-hGPR91 cells (Supplementary Information). Taken together, these results suggest that GPR91 activation by succinate couples to at least two signalling pathways, a pertussis-toxin-sensitive $G_{i} / G_{o}$ pathway and a pertussis-toxininsensitive $G_{\mathrm{q}}$ pathway. Similarly, $\alpha$-ketoglutarate stimulated inositol phosphate formation in a GPR99-dependent manner (Fig. 2g).
Because GPR99 activation did not affect cAMP levels (data not shown), and both inositol phosphate formation (Fig. 2g) and $\left[\mathrm{Ca}^{2+}\right]_{\mathrm{i}}$ flux (Supplementary Information) were found to be insensitive to pertussis toxin, GPR99 seems to act exclusively through a $\mathrm{G}_{\mathrm{q}}$-mediated pathway.

Ligand-induced receptor internalization is often characteristic of GPCR activation and signal attenuation ${ }^{9}$. Immunofluorescent staining of cells expressing Flag-tagged GPR91 or GPR99 revealed that both GPR91 and GPR99 were localized to the plasma membrane (Fig. 2h). Ligand stimulation induced the internalization of both receptors (Fig. $2 \mathrm{~h}$ ).

Because the dicarboxylate groups of succinate and $\alpha$-ketoglutarate are required for the activation of GPR91 and GPR99, we speculated that basic residues of GPR91 and GPR99 might be important in binding dicarboxylate ligands. A partial three-dimensional model of GPR91 was generated. Mutation of Arg 99, His 103, Arg 252 or Arg 281 (Supplementary Information) abolished GPR91 activation by succinate, whereas mutation of His 249 , Arg 255 or Tyr 107 had no effect on receptor function (Fig. 3a). Various GPR91 mutants were expressed at similar levels (Fig. 3b) and localized at the plasma membrane (data not shown). All four residues required for GPR91 activation by succinate (Arg 99, His 103, Arg 252 and Arg 281) are directed into the central cavity where cis-retinal binds in the rhodopsin structure (Fig. 3c, d). These four positively charged residues cluster together, Arg 99 and His 103 of helix III on one side and Arg 252 of helix VI and Arg 281 of helix VII on the other side,
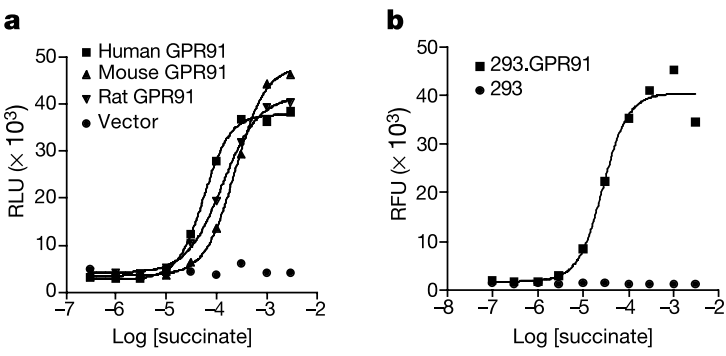

e

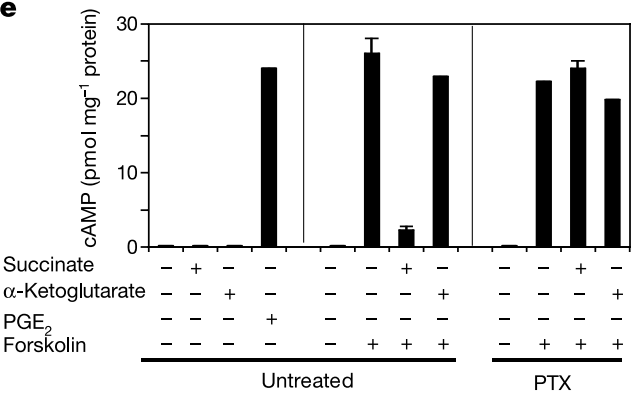

$\mathbf{f}$
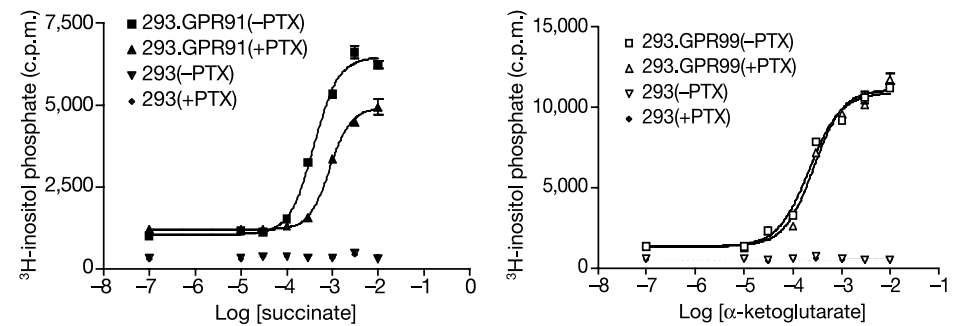

h

GPR91

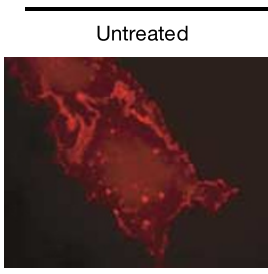

Non-permeabilized

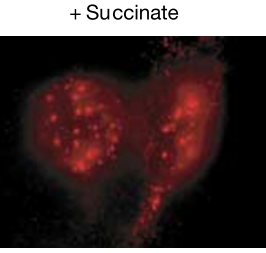

Permeabilized

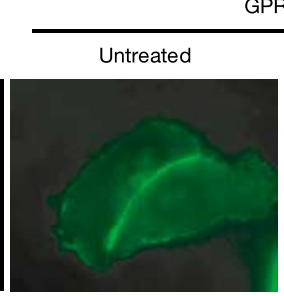

Non-permeabilized

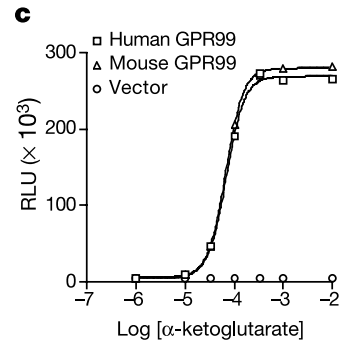

Log $[\alpha-$ ketoglutarate $]$

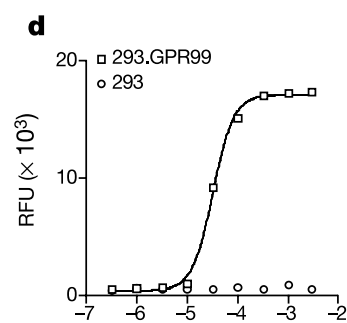

Log $[\alpha$-ketoglutarate $]$

\section{$\mathbf{g}$}

$\mathrm{KK}-\beta$

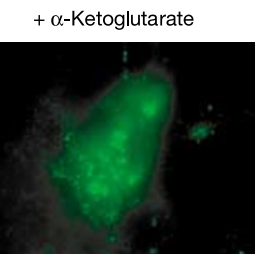

Permeabilized

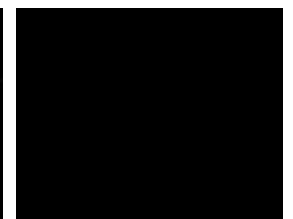

Non-permeabilized

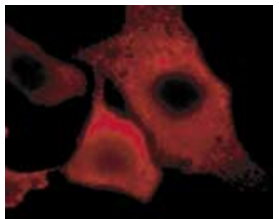

Permeabilized
Figure 2 Pharmacological characterization. Data are shown as means \pm s.e.m. for three or four determinations. a, GPR91 activation by succinate in aequorin assay. b, FLIPR assay in 293-hGPR91 cells. RFU, relative fluorescence unit. c, d, Activation of GPR99 by $\alpha$-ketoglutarate in aequorin (c) and FLIPR assays (d). e, Effect of succinate $(200 \mu \mathrm{M})$ on intracellular cAMP in 293-hGPR91 cells. Pertussis toxin and forskolin were included as indicated. Prostaglandin $\mathrm{E}_{2}\left(\mathrm{PGE}_{2}\right)$ was used as a control. f, Inositol phosphate formation in 293-hGPR91 cells and the effect of pertussis toxin (PTX). g, Inositol phosphate accumulation in 293-hGPR99 cells and the effect of PTX. h, Immunofluorescence staining. Plasma membrane localization of the receptors (in non-permeabilized cells) and the internalized vesicles (in permeabilized cells) were visualized by anti-Flag antibody. IKK- $\beta$, IкB kinase- $\beta$. An intracellular protein IKK- $\beta^{30}$ served as a control. 


\section{letters to nature}
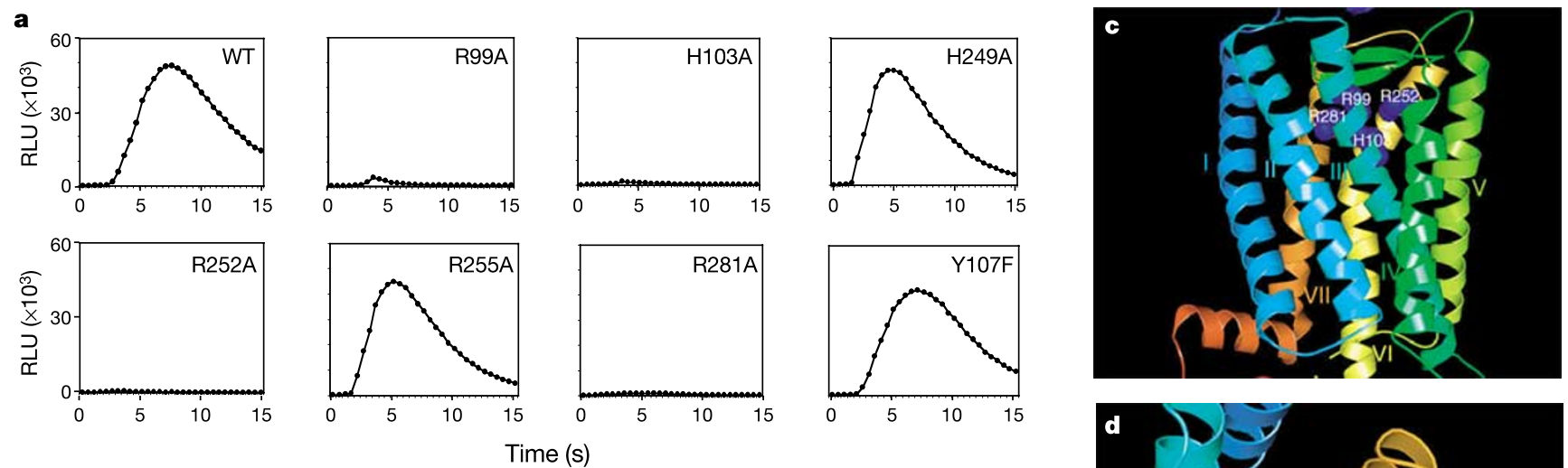

b

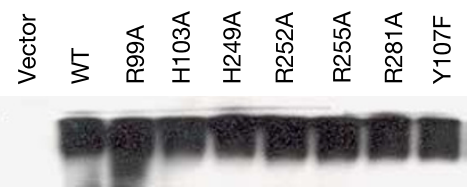

WB: Anti-Flag

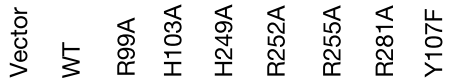

WB: Anti-tubulin

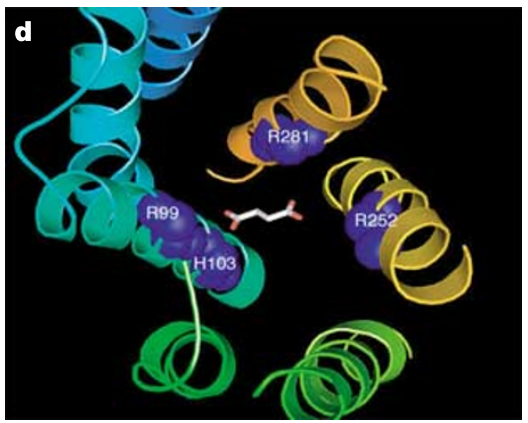

Figure 3 Mutational analysis and predicted three-dimensional model for GPR91. a, Activation of wild-type (WT) and mutant GPR91 receptors by succinate $(200 \mu \mathrm{M})$ in aequorin assay in $\mathrm{CHO}$ cells. $\mathbf{b}$, The expression of wild-type and mutant GPR91 receptors in $\mathrm{CHO}$ cells as determined by western blot (WB) analysis. c, Predicted sites for Arg 99,

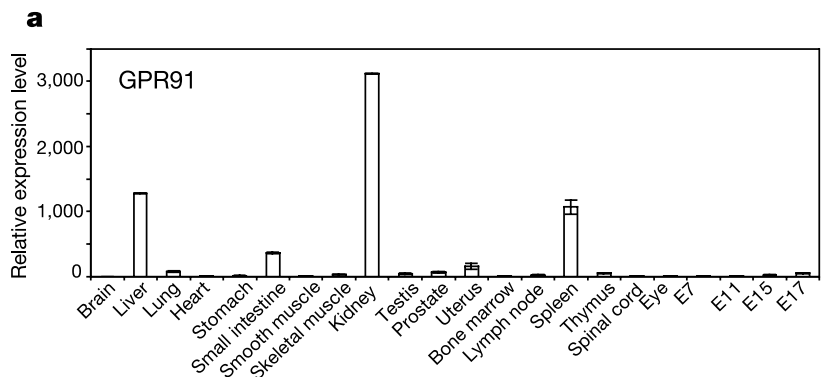

c

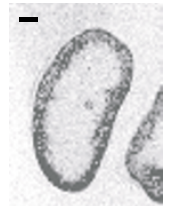

Antisense Sense

GPR91

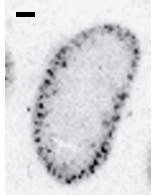

Antisense Sense

GPR99

e

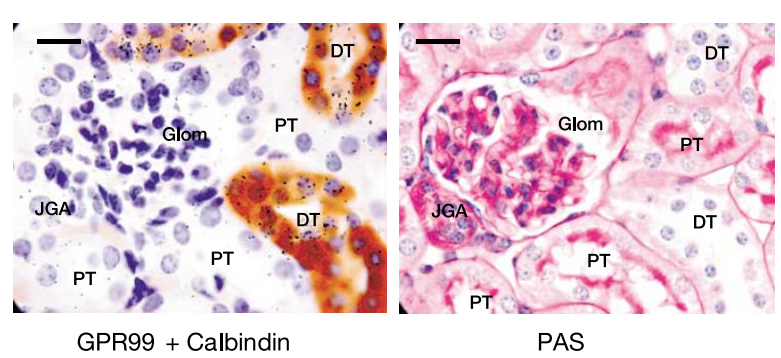

Figure 4 Regional distribution of GPR91 and GPR99. a, b, Q-RT-PCR analysis of GPR91 (a) and GPR99 (b) in mouse tissue. Data are shown as means \pm s.e.m. for triplicate determinations. c, Localization of GPR91 and GPR99 in mouse kidney sections by in situ hybridization. Scale bar, $1 \mathrm{~mm}$. d, Localization of GPR91 (black grains) in the proximal tubules. PT, proximal tubule; DT, distal tubule; glom, glomerulus. Sections were

His 103, Arg 252 and $\operatorname{Arg} 281$ in the three-dimensional ribbon presentation of GPR91. The main chains of these four residues are coloured in blue. $\mathbf{d}$, Top view from the extracellular side of the four basic residues in the ligand-binding pocket with a succinic acid molecule placed in the cavity.

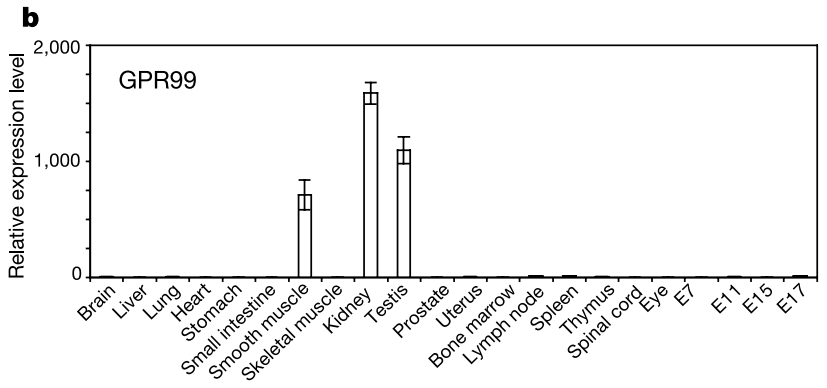

d

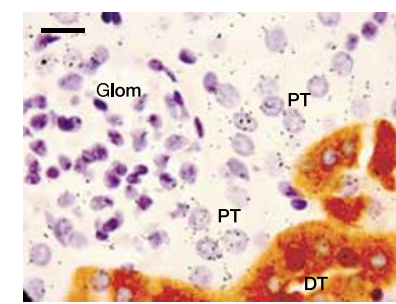

GPR91

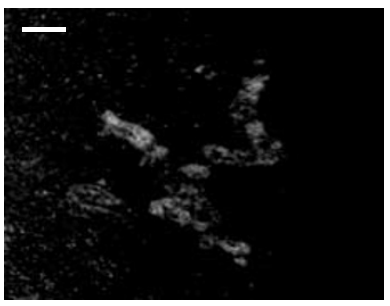

GPR99 (dark field)

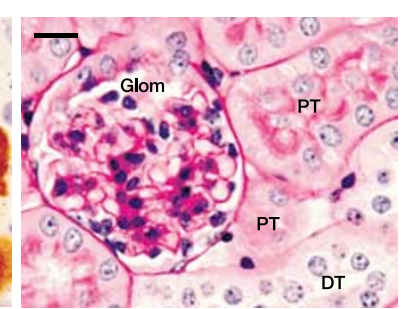

PAS prestained with anti-calbindin (brown) before hybridizing to GPR91 probe. Periodic acidSchiff (PAS) staining identified proximal tubules. Scale bar, $25 \mu \mathrm{m}$. e, f, Localization of GPR99 (black grains in $\mathbf{e}$ and white signals in $\mathbf{f}$ ) in the distal tubules. Scale bar: $25 \mu \mathrm{m}(\mathbf{e})$; $0.25 \mathrm{~mm}(\mathbf{f})$. 
and may provide a unique electrostatic complementary binding environment for succinate (Fig. 3d). A comparison of about 300 family A GPCRs revealed that GPR91 and GPR99 uniquely contain all four basic residues, suggesting that GPR91 and GPR99 are specialized receptors for dicarboxylate ligands.

Expression analysis by quantitative reverse-transcriptasemediated polymerase chain reaction (Q-RT-PCR) revealed that the GPR91 and GPR99 messenger RNAs were each predominantly expressed in the kidney, with limited expression in other tissues (GPR91 in the liver and the spleen, and GPR99 in the testis and the smooth muscle; Fig. 4a, b and refs 3, 4). In situ hybridization experiments indicated specific GPR91 and GPR99 expression in the cortical region of the mouse kidney (Fig. 4c). GPR91 was detected mainly in the proximal tubules, as demonstrated by the presence of a prominent brush border projecting into the lumen on periodicacid-Schiff-stained adjacent sections (Fig. 4d). GPR91 was also found, to a smaller extent, in the distal tubules (revealed by staining with antibodies against the distal-tubule marker calbindin ${ }^{10} ;$ Fig. $4 \mathrm{~d}$ ) and the juxtaglomerular apparatus (Supplementary Information). In contrast, GPR99 was found predominantly in the distal tubules (Fig. 4e, f).

Intravenous injection of succinate into Sprague-Dawley rats increased plasma renin activity (Fig. 5a), which is consistent with a previous report that succinate-treated kidney cultures ex vivo released $\operatorname{renin}^{11}$. Renin is a key enzyme in the renin-angiotensin system, which is essential in blood pressure regulation ${ }^{12,13}$. Cumulative intravenous administration of succinate produced a dosedependent increase in mean arterial pressure (MAP) (Fig. 5b). Bilateral nephrectomy completely abolished this effect (Fig. 5c), demonstrating the kidney as the primary site of action. To dissect the pharmacological mechanism for succinate-induced hypertension, the angiotensin-converting enzyme inhibitor captopril was used. Pretreatment with captopril significantly attenuated the hypertensive effect of succinate (Fig. 5d), indicating that the angiotensin-converting enzyme product(s) might mediate hypertension induced by succinate. In contrast, neither nephrectomy nor captopril treatment had any effect on angiotensin II-induced hypertension (Fig. 5c, d).

To further explain the mechanisms underlying succinate-induced hypertension, we generated GPR91-deficient mice by homologous recombination (Fig. 5e, f). Mice homozygous for the targeted allele of GPR91 are viable and have no discernable phenotype. The systolic blood pressures and heart rates were similar in unanaesthetized wild-type and GPR91-deficient mice (Fig. 5g; systolic blood pressures were $129.5 \pm 1.5 \mathrm{mmHg}$ for wild-type and $131.2 \pm$ $2.4 \mathrm{mmHg}$ for GPR91-deficient mice; heart rates were $613 \pm 12.4$ beats $\min ^{-1}$ for wild-type and $606 \pm 11.4$ beats $\min ^{-1}$ for GPR91deficient mice). However, succinate could no longer induce hypertension in GPR91-deficient mice (Fig. 5h, i). In contrast, angiotensin II increased blood pressure similarly in wild-type and

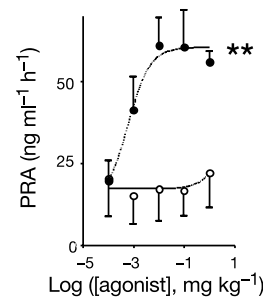

b

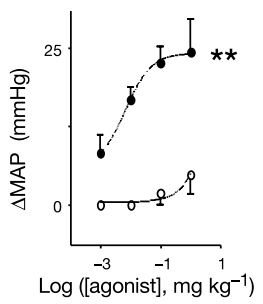

c

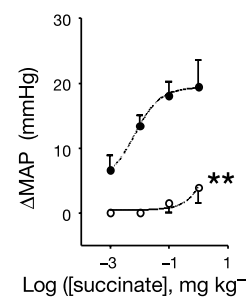

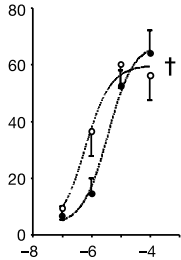

Log ([Angll], $\left.\mathrm{mg} \mathrm{kg}^{-1}\right)$
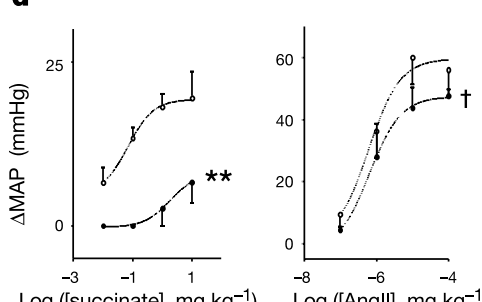

Log ([succinate], $\left.\mathrm{mg} \mathrm{kg}^{-1}\right) \quad$ Log ([Angll], $\mathrm{mg} \mathrm{kg}^{-1}$ )
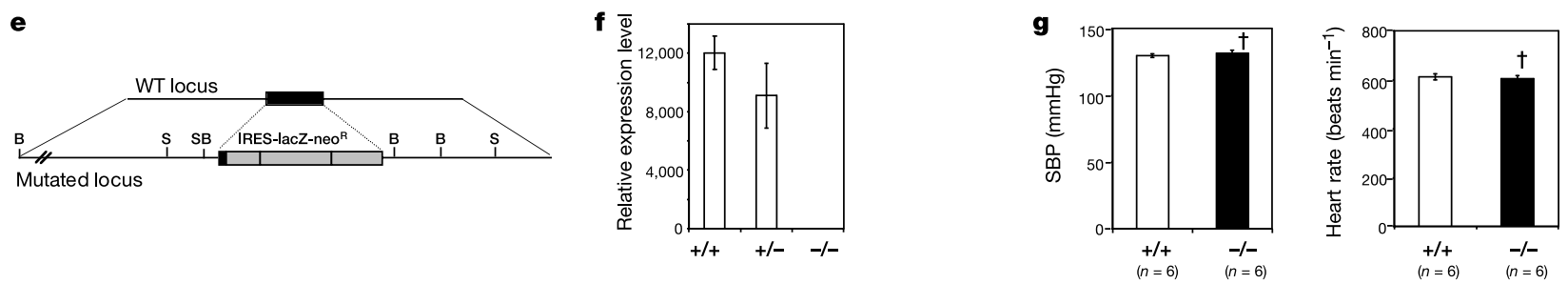
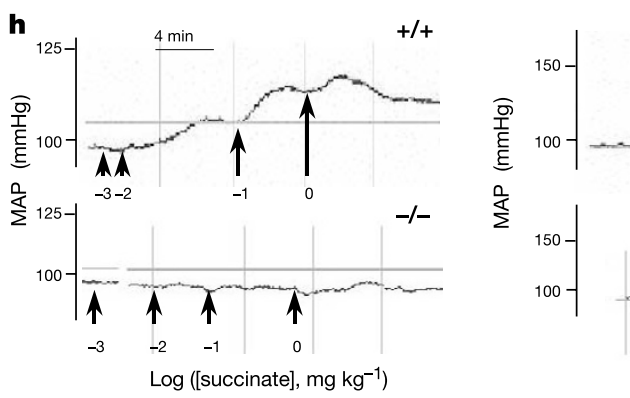

i

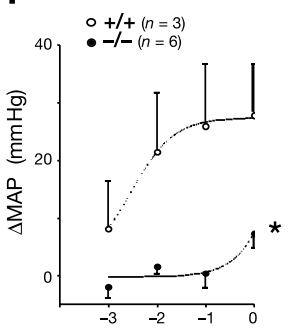

Log ([succinate], $\mathrm{mg} \mathrm{kg}^{-1}$ )

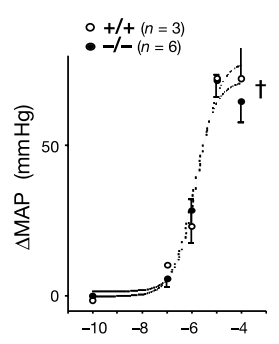

Log ([Angll], $\mathrm{mg} \mathrm{kg}^{-1}$ )
Figure 5 The pro-hypertensive effect of succinate and GPR91. All results are shown as mean \pm s.e.m. Single asterisk, $P<0.05$; double asterisk, $P<0.01$; dagger, $P>0.05$ (two-way analysis of variance or $t$-test). a, Plasma renin activity (PRA) in Sprague-Dawley rats by intravenous succinate infusion. Filled circles, succinate $(n=6)$; open circles, $\alpha$-ketoglutarate $(n=6)$. b. MAP in Sprague-Dawley rats by intravenous succinate infusion. Values are the change in MAP $(\triangle M A P, m m H g)$ for each test group above its vehicle-injected control group. Filled circles, succinate $(n=8)$; open circles, $\alpha$-ketoglutarate $(n=6)$. c, Effect of bilateral nephrectomy. Filled circles, kidney intact $(n=6)$; open circles, bilateral nephrectomy $(n=4)$. d, Effect of captopril. Filled circles, captopril present $(n=4)$; open circles, captopril absent $(n=6)$. e. Schematic map of the GPR91 wild-type (WT) locus and the inactivated GPR91 allele. Black box, open reading frame of GPR91 gene; S, Spel; B, BamHl; lacZ, $\beta$-galactosidase expression cassette; neo ${ }^{R}$, neomycin resistance gene cassette. f, Q-RT-PCR analysis of GPR91 mRNA in kidneys of wild type (+/+), GPR91-heterozygous (+/-) and GPR91-deficient (-/-) mice. $\mathbf{g}$, Systolic blood pressure and heart rate of unanaesthetized wild-type and GPR91-deficient mice. h, i, MAP tracing measurements (h) and dose-response curves (i) of wild-type and GPR91-deficient mice administered intravenously with succinate (left panels) or angiotensin II (Angll) (right panels). 
GPR91-deficient mice (Fig. 5h, i), indicating that the hypertensive effect of succinate might be mediated by GPR91.

Succinate and $\alpha$-ketoglutarate are normally present in mitochondria, but are found in circulation with mean plasma levels of about $5 \mu \mathrm{M}$ for succinate and about $25 \mu \mathrm{M}$ for $\alpha$-ketoglutarate ${ }^{14-16}$. Succinate is known to increase the reabsorption of phosphate and glucose into the proximal tubule ${ }^{17}$ and to stimulate gluconeogenesis $^{18}$. Accumulation of extracellular succinate is observed in physiological and/or pathophysiological states linked to the deterioration of blood supply, such as ischaemia ${ }^{19,20}$. It is known that the renovascular hypertension is often associated with restricted blood supply to the kidney owing to the obstruction of renal arteries in diseases such as atherosclerosis ${ }^{5}$. It is possible that the accumulation of succinate in ischaemic conditions might contribute to stenosisassociated hypertension through the activation of GPR91. Although we have shown that the hypertensive effect of succinate is mediated by GPR91 through the activation of the renin-angiotensin system, the molecular mechanism of renin release by succinate is not clear. Because GPR91 is a $\mathrm{G}_{\mathrm{q}^{-}}$and $\mathrm{G}_{\mathrm{i}}$-coupled receptor, it is unlikely that GPR91 functions directly on the juxtaglomerular cells to stimulate renin release, which is usually triggered by $G_{s}$ activation ${ }^{21}$. It remains to be investigated whether sympathetic nerve activity, prostaglandins or nitric oxide ${ }^{22}$ might provide an indirect mechanism for succinate-induced renin release. The presence of GPR91 in the liver and the spleen might also have functions in mediating these effects.

Thus, we have identified succinate as the natural ligand for GPR91, and $\alpha$-ketoglutarate as the ligand for the homologous receptor GPR99. We have shown that intermediates in the citric acid cycle function as signalling molecules, engendering renewed interest in a biochemical pathway discovered more than 60 years ago. We have provided evidence that the pro-hypertensive effect of succinate is mediated by GPR91 and involves the renin-angiotensin system. GPR91 activation by succinate might therefore provide a link between energy homeostasis/metabolic status and haemodynamic regulation. The finding that intermediates in the citric acid cycle are GPCR ligands should facilitate the understanding of molecular links of the citric acid cycle to metabolic diseases, such as hypertension, atherosclerosis and diabetes, and the design of novel drugs with GPR91 and GPR99 as molecular targets.

\section{Methods}

\section{Purification of GPR91 ligand}

Pig kidneys (6 kg; Pel-Freeze) were homogenized and extracted in ethanol/water/acetic acid (50/46/4, by vol.). The supernatant was freeze-dried, resuspended in $10 \mathrm{mM} \mathrm{K}_{2} \mathrm{HPO}_{4}$ $\mathrm{pH}$ 8.0, applied to an XK50/20 Q Sepharose anion-exchange column (Amersham), and developed with a $0-0.25 \mathrm{M} \mathrm{NaCl}$ linear gradient. The active fractions were subjected to three more steps of purification: a Hiload 26/60 Superdex30 size-exclusion column (developed in Hank's buffered saline at pH 7.35); a Superdexpeptide HR10/30 sizeexclusion column (developed in $0.1 \%$ trifluoroacetic acid/water); and a $\mathrm{C}_{18}$

$4.6 \mathrm{~mm} \times 250 \mathrm{~mm}$ reverse-phase column (developed in $0.1 \%$ trifluoroacetic acid /water) The active fractions from the final purification step were freeze-dried and dissolved in ${ }^{2} \mathrm{H}_{2} \mathrm{O}$ for NMR and mass spectrometry analysis.

\section{NMR and mass spectrometry}

The ${ }^{1} \mathrm{H}$ and ${ }^{13} \mathrm{C}$ NMR spectra were recorded on a Bruker Avance DRX700 spectrometer (Bruker Analytik $\mathrm{GmbH}$ ) at 700 and $176 \mathrm{MHz}$, respectively. The mass spectrum was obtained on an HP 1100MSD spectrometer (Agilent Technologies) with electrospray ionization in positive detection mode. A full-scan mass spectrum covering the $m / z$ range 50-500 was acquired.

\section{Cell culture and signalling assays}

Chinese hamster ovary $(\mathrm{CHO})$ cells were transiently transfected with $5 \mu \mathrm{g}$ GPCR and $5 \mu \mathrm{g}$ aequorin reporter gene $\mathrm{e}^{7}$ in $100-\mathrm{mm}$ dishes as described ${ }^{23}$. Aequorin luminescence was recorded with a Microlumat (Berthold). FLIPR assays were performed on FLIPR384 with a FLIPR calcium assay kit (Molecular Devices). Inositol phosphate formation was assayed as the incorporation of ${ }^{3} \mathrm{H}$-myo-inositol as described ${ }^{23,24}$. Cells were stimulated with compounds in the presence of $10 \mathrm{mM} \mathrm{LiCl}$ for $40 \mathrm{~min}$ at $37^{\circ} \mathrm{C}$ before the addition of $20 \mathrm{mM}$ formic acid. cAMP assay was performed with the cAMP-Screen system (Applied Biosystems). Cells were stimulated for $20 \mathrm{~min}$ with $10 \mu \mathrm{M}$ forskolin before treatment with compounds for a further $20 \mathrm{~min}$ at $37^{\circ} \mathrm{C}$. Anti-phospho-Erk and anti-Erk monoclonal antibodies were obtained from New England Biolabs. Anti-Flag and anti-tubulin antibodies were obtained from Sigma. Western blots were detected with the ECL Plus detection system (Amersham). For some studies, $100 \mathrm{ng} \mathrm{ml}^{-1}$ pertussis toxin (Calbiochem) was incubated with cells for $16 \mathrm{~h}$.

\section{Immunofluorescence staining}

Human embryonic kidney 293 cells transfected with Flag-tagged GPR91 or GPR99 were plated on poly-(D-lysine)-treated slides. Cell-surface staining and ligand-induced internalization was performed as described ${ }^{9}$. Internalization of GPR91 or GPR99 was induced for $10 \mathrm{~min}$ by succinate $(200 \mu \mathrm{M})$ or $\alpha$-ketoglutarate $(300 \mu \mathrm{M})$ at $37^{\circ} \mathrm{C}$.

\section{In situ hybridization, immunohistochemistry and expression analysis}

These were performed as described ${ }^{10,25} \cdot\left[{ }^{33} \mathrm{P}\right]$ UTP-labelled antisense or sense RNA probes for mouse GPR91, GPR99 or renin were hybridized to paraformaldehyde-fixed, paraffinembedded mouse kidney sections. For periodic acid-Schiff and renin staining, adjacent mouse kidney sections were used. For co-localization with calbindin on the same tissue section, slides were incubated with anti-calbindin antibody (Sigma) and developed with Vectastain avidin-biotin-complex kit (Vector Lab) with 3,3'-diaminobenzidine as substrate before hybridization in situ. Sections were counterstained with haematoxylin or toluidine blue. Q-RT-PCR was performed on an ABI Prism 7700 sequence detector using Taqman Gold kit (Applied Biosystems). Ratios of GPR91 or GPR99 to glyceraldehyde-3phosphate dehydrogenase mRNA were calculated. Primer and probe sequences for GPR91 and GPR99 are available from the authors on request.

\section{Receptor model}

The published bovine rhodopsin structure was used as a template for generating a partial GPR91 structure model ${ }^{26}$. The GPR91 primary sequence was aligned to bovine rhodopsin (PDB code 1F88; Supplementary Information), and the three-dimensional homology model was generated with the Modeller program.

\section{In vivo experiments}

All experiments were performed under protocols approved by the Institutional Animal Care and Use Committee. Animals were housed in a temperature-controlled room under a $12 \mathrm{~h}$ light-dark cycle with water and standard rodent chow available ad libitum. Blood pressure was measured in unanaesthetized animals by the tail-cuff method or in pentobarbitol-anaesthetized animals by the intra-arterial method for those involving chemical administration ${ }^{27,28}$. Male Sprague-Dawley rats (about $300 \mathrm{~g}$; Harlan) or mice (about $30 \mathrm{~g}$; Lexicon) were used. Systolic blood pressure was recorded in mice for 5 days; at least 20 measurements were made daily with a BP-2000 blood pressure analysis tail-cuff system (Visitech Systems). The mean systolic blood pressure and pulse rate were taken for each animal. Intra-arterial measurement of blood pressure was achieved with a polyethylene PE50 (for rats) or a stretched PE10 (for mice) catheter implanted into the carotid artery, with another implanted into the jugular vein before the experiments. The artery catheter was connected to a high-sensitivity isometric transducer (Harvard Apparatus) for continuous recording of blood pressure. MAP data were acquired by Ponemch Physiology Platform (Gould Instrument). The venous catheter was used for chemical administration. Bilateral nephrectomy was performed as described ${ }^{29}$. Captopril $\left(10 \mathrm{mg} \mathrm{kg}^{-1}\right.$ ) was given as a bolus injection $30 \mathrm{~min}$ before the administration of succinate. Plasma renin activity was determined by radioimmunoassay as the rate of angiotensin I generation (DiaSorin). GPR91-deficient mice were generated by homologous recombination in embryonic stem cells (Lexicon). Results are expressed as means \pm s.e.m. Statistical significance was evaluated by Student's $t$-test or two-way analysis of variance, followed by Dunnett's post-hoc test. Differences resulting in $P<0.05$ were considered significant.

Received 3 February; accepted 15 March 2004; doi:10.1038/nature02488.

1. Krebs, H. A. The history of the tricarboxylic acid cycle. Perspect. Biol. Med. 14, 154-170 (1970).

2. Eng, C., Kiuru, M., Fernandez, M. J. \& Aaltonen, L. A. A role for mitochondrial enzymes in inherited neoplasia and beyond. Nature Rev. Cancer 3, 193-202 (2003).

3. Wittenberger, T., Schaller, H. C. \& Hellebrand, S. An expressed sequence tag (EST) data mining strategy succeeding in the discovery of new G-protein coupled receptors. J. Mol. Biol. 307, 799-813 (2001)

4. Wittenberger, T. et al. GPR99, a new G protein-coupled receptor with homology to a new subgroup of nucleotide receptors. BMC Genomics 3, 17-22 (2002).

5. Olin, J. W. Atherosclerotic renal artery disease. Cardiol. Clin. 20, 547-562 (2002).

6. Jandeleit-Dahm, K. \& Cooper, M. E. Hypertension and diabetes. Curr. Opin. Nephrol. Hypertens. 11, 221-228 (2002).

7. Stables, J. et al. A bioluminescent assay for agonist activity at potentially any G-protein-coupled receptor. Anal. Biochem. 252, 115-126 (1997).

8. Lee, D. K. et al. Discovery and mapping of ten novel G protein-coupled receptor genes. Gene $\mathbf{2 7 5}$, 83-91 (2001).

9. von Zastrow, M. \& Kobilka, B. K. Antagonist-dependent and -independent steps in the mechanism of adrenergic receptor internalization. J. Biol. Chem. 269, 18448-18452 (1994).

10. Campean, V., Kricke, J., Ellison, D., Luft, F. C. \& Bachmann, S. Localization of thiazide-sensitive $\mathrm{Na}^{+}-\mathrm{Cl}^{-}$cotransport and associated gene products in mouse DCT. Am. J. Physiol. Renal Physiol. 281, F1028-F1035 (2001).

11. Baumbach, L., Leyssac, P. P. \& Skinner, S. L. Studies on renin release from isolated superfused glomeruli: effects of temperature, urea, ouabain and ethacrynic acid. J. Physiol. (Lond.) 258, 243-256 (1976).

12. Lavoie, J. L. \& Sigmund, C. D. Minireview: overview of the renin-angiotensin system-an endocrine and paracrine system. Endocrinology 144, 2179-2183 (2003).

13. Gurley, S. B., Le, T. H. \& Coffman, T. M. Gene-targeting studies of the renin-angiotensin system: mechanisms of hypertension and cardiovascular disease. Cold Spring Harb. Symp. Quant. Biol. 67, 451-457 (2002). 
14. Bergmeyer, H. U. Methods of Enzymatic Analysis (Verlag Chemie, Weinheim/Bergstr., 1974).

15. Kushnir, M. M., Komaromy-Hiller, G., Shushan, B., Urry, F. M. \& Roberts, W. L. Analysis of dicarboxylic acids by tandem mass spectrometry. High-throughput quantitative measurement of methylmalonic acid in serum, plasma, and urine. Clin. Chem. 47, 1993-2002 (2001).

16. Martin, M., Ferrier, B. \& Baverel, G. Transport and utilization of alpha-ketoglutarate by the rat kidney in vivo. Pflugers Arch. 413, 217-224 (1989).

17. Gullans, S. R., Kone, B. C., Avison, M. J. \& Giebisch, G. Succinate alters respiration, membrane potential, and intracellular $\mathrm{K}^{+}$in proximal tubule. Am. J. Physiol. 255, F1170-F1177 (1988).

18. Gullans, S. R., Brazy, P. C., Dennis, V. W. \& Mandel, L. J. Interactions between gluconeogenesis and sodium transport in rabbit proximal tubule. Am. J. Physiol. 246, F859-F869 (1984).

19. Krebs, H. A. Rate control of the tricarboxylic acid cycle. Adv. Enzyme Regul. 8, 335-353 (1970)

20. Hems, D. A. \& Brosnan, J. T. Effects of ischaemia on content of metabolites in rat liver and kidney in vivo. Biochem. J. 120, 105-111 (1970).

21. Pan, L. et al. Critical roles of a cyclic AMP responsive element and an E-box in regulation of mouse renin gene expression. J. Biol. Chem. 276, 45530-45538 (2001)

22. Hackenthal, E., Paul, M., Ganten, D. \& Taugner, R. Morphology, physiology, and molecular biology of renin secretion. Physiol. Rev. 70, 1067-1116 (1990)

23. An, S. et al. Identification and characterization of a melanin-concentrating hormone receptor. Proc Natl Acad. Sci. USA 98, 7576-7581 (2001)

24. Brandish, P. E., Hill, L. A., Zheng, W. \& Scolnick, E. M. Scintillation proximity assay of inositol phosphates in cell extracts: high-throughput measurement of G-protein-coupled receptor activation. Anal. Biochem. 313, 311-318 (2003).

25. Chuang, P. T., Kawcak, T. \& McMahon, A. P. Feedback control of mammalian Hedgehog signaling by the Hedgehog-binding protein, Hipl, modulates Fgf signaling during branching morphogenesis of the lung. Genes Dev. 17, 342-347 (2003).

26. Palczewski, K. et al. Crystal structure of rhodopsin: A G protein-coupled receptor. Science 289, 739-745 (2000)

27. Krege, J. H., Hodgin, J. B., Hagaman, J. R. \& Smithies, O. A noninvasive computerized tail-cuff system for measuring blood pressure in mice. Hypertension 25, 1111-1115 (1995)

28. Sugiyama, F. et al. QTL associated with blood pressure, heart rate, and heart weight in $\mathrm{CBA} / \mathrm{CaJ}$ and BALB/cJ mice. Physiol. Genomics 10, 5-12 (2002).

29. Silva, A. P. et al. Bilateral nephrectomy delays gastric emptying of a liquid meal in awake rats. Ren. Fail. 24, 275-284 (2002)

30. Woronicz, J. D., Gao, X., Cao, Z., Rothe, M. \& Goeddel, D. V. ІкB kinase- $\beta$ : NF-кB activation and complex formation with IkB kinase- $\alpha$ and NIK. Science 278, 866-869 (1997).

Supplementary Information accompanies the paper on www.nature.com/nature

Acknowledgements We thank J. Reagan for critical comments during ligand identification; L. Yang for sharing reagents for in situ hybridization; G. Cutler, J. Knop, H. Baribault, J. Ma, S.-C. Miao, W. Inman, C. Ogden, S. Shuttleworth and M. Rich for providing support and discussions; and D. Goeddel, B. Lemmon and T. Hoey for critical reading of the manuscript.

Competing interests statement The authors declare that they have no competing financial interests.

Correspondence and requests for materials should be addressed to L.L. (ling@tularik.com).

\section{Aquaporin-0 membrane junctions reveal the structure of a closed water pore}

\section{Tamir Gonen ${ }^{1}$, Piotr Sliz ${ }^{2}$, Joerg Kistler ${ }^{3}$, Yifan Cheng ${ }^{1} \&$ Thomas Walz $^{1}$}

${ }^{1}$ Department of Cell Biology, Harvard Medical School, 240 Longwood Avenue, Boston, Massachusetts 02115, USA

${ }^{2}$ Howard Hughes Medical Institute and Children's Hospital Laboratory of Molecular Medicine, 320 Longwood Avenue, and Department of Biological Chemistry and Molecular Pharmacology, Harvard Medical School, 240 Longwood Avenue, Boston, Massachusetts 02115, USA

${ }^{3}$ School of Biological Sciences, University of Auckland, Auckland PO Box 92019, New Zealand

The lens-specific water pore aquaporin-0 (AQP0) is the only aquaporin known to form membrane junctions in vivo ${ }^{1}$. We show here that AQP0 from the lens core, containing some carboxyterminally cleaved $\mathrm{AQPO}^{2,3}$, forms double-layered crystals that recapitulate in vivo junctions. We present the structure of the AQP0 membrane junction as determined by electron crystallography. The junction is formed by three localized interactions between AQP0 molecules in adjoining membranes, mainly mediated by proline residues conserved in AQP0s from different species but not present in most other aquaporins. Whereas all previously determined aquaporin structures show the pore in an open conformation ${ }^{4-9}$, the water pore is closed in AQP0 junctions. The water pathway in AQP0 also contains an additional pore constriction, not seen in other known aquaporin structures $^{4-9}$, which may be responsible for pore gating.

AQP0 is a member of the aquaporin family, members of which form pores that are either highly selective for water (aquaporins) or also permeable to other small neutral solutes such as glycerol (aquaglyceroporins) (reviewed in ref. 10). To date, the atomic structures of three aquaporins have been determined (AQP1 ${ }^{4-6}$, $\mathrm{GlpF}^{7,8}$ and $\mathrm{AQPZ}{ }^{9}$ ). Sequence alignment shows AQP0 to be closely related to the pure water channel AQP1 (43.6\% identity, $62.6 \%$ similarity). The presence of His 172 , a residue conserved only in aquaporins but substituted in aquaglyceroporins, also suggests that AQP0 forms a pure water pore. AQP0 water permeability at neutral $\mathrm{pH}$ is approximately 40 times lower than that of $\mathrm{AQP} 1^{11}$, but AQP0 water conductance doubles under mildly acidic conditions ${ }^{12}$. In the case of aquaporins in plant roots, a $\mathrm{pH}$-dependent closure of the water pores has been reported ${ }^{13}$. Thus, evidence suggests that certain aquaporin pores are gated.

AQP0 water pores are considered essential for the lens microcirculation system, proposed to supply deeper-lying fibre cells with nutrients and to clear waste products ${ }^{14,15}$. Unlike all other aquaporins, AQP0 is also present in membrane junctions. It is particularly enriched in the $11-13 \mathrm{~nm}$ thin junctions between lens fibre cells, that feature square AQP0 arrays ${ }^{1}$. Atomic force microscopy analysis of in vitro reconstituted AQP0 two-dimensional crystals demonstrated these crystals to be double-layered ${ }^{16}$.

Using AQP0 from the core of sheep lenses, where some of the AQP0 is proteolytically cleaved near the $C$ terminus at various sites in an age-dependent manner ${ }^{2,3}$, we reproduced the double-layered two-dimensional crystals ${ }^{16,17}$. When core AQP0 was reconstituted at a lipid-to-protein ratio of $0.25(\mathrm{w} / \mathrm{w})$, large membrane sheets formed $(>6 \mu \mathrm{m})$ that in some cases showed two parallel edges, revealing them to be double-layered (Fig. 1a). The crystals showing $p 422$ symmetry had lattice constants of $a=b=65.5 \AA$ and a thickness of $11 \mathrm{~nm}$ (Fig. 2a), the same dimensions as thin junctions in the lens ${ }^{1}$. Double-layered AQP0 two-dimensional crystals are therefore likely to recapitulate thin lens fibre cell junctions.

Electron diffraction analysis of AQP0 crystals (tilted to an angle of up to $70^{\circ}$ ) produced strong diffraction spots to $3 \AA$ resolution in all directions (Fig. 1b, c; the electron crystallographic data are summarized in Table 1). As the crystal structure of the homologous bovine AQP1 was available ${ }^{5}$, we determined the structure of the AQP0 membrane junction by molecular replacement, thus avoiding the cumbersome and time-consuming process of collecting highresolution images of tilted specimens. Sequencing of cloned sheep AQP0 showed an identical amino acid sequence to bovine AQP0 ${ }^{18}$, with the exception of three conservative (S20T, M90V and S240T) and one non-conservative substitutions $(\mathrm{C} 14 \mathrm{~F})$.

Our model (Fig. 2a) shows unique features that enable AQP0 to form membrane junction interactions. These differ from those previously suggested based on atomic force microscopy data ${ }^{16}$. The extracellular surface of AQP0 is rather flat and the interactions are mediated by direct contacts of the corresponding loops in the opposing AQP0 molecules (Fig. 2a). Loop C, connecting $\alpha$-helices three and four, is significantly shorter than in AQP1 and GlpF. The shortened loop C (also seen in AQP2, AQP5, AQP6 and AQP8) is crucial for the formation of the very tight AQP0 junction, as it allows three specific interactions to be formed that are mediated almost exclusively by proline residues.

The most striking interaction involves Pro 38, in extracellular loop A. The proline residues (Pro 38) from eight symmetry-related AQP0 molecules in the stacked tetramers come together to form a 